\title{
Calculating Emittance for \\ Gaussian and Non-Gaussian Distributions \\ by the Method of Correlations for Slits
}

\author{
Cheng-Yang Tan \\ Accelerator Division/Tevatron
}

\begin{abstract}
One common way for measuring the emittance of an electron beam is with the slits method. The usual approach for analyzing the data is to calculate an emittance that is a subset of the parent emittance. This paper shows an alternative way by using the method of correlations which ties the parameters derived from the beamlets to the actual parameters of the parent emittance. For parent distributions that are Gaussian, this method yields exact results. For nonGaussian beam distributions, this method yields an effective emittance that can serve as a yardstick for emittance comparisons.
\end{abstract}




\section{INTRODUCTION}

The slit method is a common way for measuring the transverse emittance of an electron beam in one selected plane. This method has been well documented and analyzed by Zhang ${ }^{1}$. However, his analysis reveals only the "subset emittance" from the data and not the beam's full emittance. ${ }^{\dagger}$ So, to get a better handle on the actual or parent emittance, the method based on correlations can be used to reveal the essential parameters for calculating the rms emittance. The idea is that the parameters calculated from the beamlets which sample the beam are correlated to the parameters of the actual emittance. And we will show that for Gaussian distributions, the method of correlations gives exact results. This method is well documented in Whittaker ${ }^{2}$, Chapter XII and we will use most of the ideas in there for our calculation.

We start off by asking the following two questions: what is the probability that a particle has a position which lies in the interval $x$ to $x+d x$ ? And what is the probability that this particle whose position is in $x$ to $x+d x$ also has a divergence which lies between $x^{\prime}$ to $x^{\prime}+d x^{\prime} ?$

We answer the two questions by first supposing that the phase space coordinate of a randomly chosen particle is $\left(x_{i}, x_{i}^{\prime}\right)$. Then the probability $h$ that $x_{i}$ has a value between $x$ and $x+d x$ is $h=f(x) d x$ where $f(x)$ is the normalized frequency distribution of the particles in $x$. This answers the first question.

Next, if $k$ is the probability that any particle between $x$ and $x+d x$ has a divergence $x_{i}^{\prime}$ which lies between $x^{\prime}$ and $x^{\prime}+d x^{\prime}$ then $k=g\left(x, x^{\prime}\right) d x^{\prime}$ where $g\left(x, x^{\prime}\right)$ is the normalized distribution function in $x^{\prime}$ which can also depend on $x$. Finally, from Fermat's Principle

$\dagger$ A quote from the Introduction of Zhang's paper: "It has to be made clear that a measured emittance is not beam's real mathematical emittance because of the following two reasons: Firstly, the measured emittance is based on a subset of particles instead of the whole beam ... So normally a measured emittance is at most an estimation of the real beam emittance." 
of Conjunctive Probability, the probability that a randomly selected particle has position which lies in $x$ to $x+d x$ and a divergence which lies in $x^{\prime}$ to $x^{\prime}+d x^{\prime}$ is $h k$, i.e.

$$
\phi\left(x, x^{\prime}\right) d x d x^{\prime}=f(x) g\left(x, x^{\prime}\right) d x d x^{\prime}
$$

where we have introduced the symbol $\phi\left(x, x^{\prime}\right)$ which is the normalized frequency distribution of the particles in phase space or what we usually call "the ellipse". This answers the second question. Clearly, if $g\left(x, x^{\prime}\right)$ is dependent on $x$ we have a phase space distribution that is tilted. If $g\left(x, x^{\prime}\right)$ is independent of $x$, then the distribution must be axes symmetric.

The goal is to determine $\phi\left(x, x^{\prime}\right)$ from the beamlets data. Once $\phi\left(x, x^{\prime}\right)$ is found, then it is trivial for us to calculate the rms emittance $\epsilon$ which is

$$
\epsilon\left(x, x^{\prime}\right)=\sqrt{\left\langle x^{2}\right\rangle\left\langle x^{\prime 2}\right\rangle-\left\langle x x^{\prime}\right\rangle^{2}}
$$

where

$$
\begin{aligned}
\left\langle x^{2}\right\rangle & =\int_{-\infty}^{\infty} \int_{-\infty}^{\infty} x^{2} \phi\left(x, x^{\prime}\right) d x d x^{\prime}-\left[\int_{-\infty}^{\infty} \int_{-\infty}^{\infty} x \phi\left(x, x^{\prime}\right) d x, d x^{\prime}\right]^{2} \\
\left\langle x^{\prime 2}\right\rangle= & \int_{-\infty}^{\infty} \int_{-\infty}^{\infty} x^{\prime 2} \phi\left(x, x^{\prime}\right) d x d x^{\prime}-\left[\int_{-\infty}^{\infty} \int_{-\infty}^{\infty} x^{\prime} \phi\left(x, x^{\prime}\right) d x, d x^{\prime}\right]^{2} \\
\left\langle x x^{\prime}\right\rangle= & \int_{-\infty}^{\infty} \int_{-\infty}^{\infty} x x^{\prime} \phi\left(x, x^{\prime}\right) d x d x^{\prime} \\
& -\left[\int_{-\infty}^{\infty} \int_{-\infty}^{\infty} x \phi\left(x, x^{\prime}\right) d x, d x^{\prime}\right]\left[\int_{-\infty}^{\infty} \int_{-\infty}^{\infty} x^{\prime} \phi\left(x, x^{\prime}\right) d x, d x^{\prime}\right]
\end{aligned}
$$

If $x$ and $x^{\prime}$ are uncorrelated, then $\phi\left(x, x^{\prime}\right)$ is separable and thus $\left\langle x x^{\prime}\right\rangle \equiv 0$. 


\section{SETUP}

The usual setup for measuring emittance with beam slits is shown in Figure 1 . The distance between the slits and the screen is $L$. The particles from each slit start diverging when travelling between the slit and screen. The particles hit the screen and produce an intensity distribution that is proportional to the number of particles at that point. If there are no axis offsets between the slits and the screen and the distance $L$ is chosen so that the intensity on the screen does not overlap, then we can associate each distinct intensity strip with its corresponding slit. The divergence $x_{j}^{\prime}$ from slit $x_{s i}$ and image position $X_{j}$ in that strip is

$$
x_{j}^{\prime}=\frac{X_{j}-x_{s i}}{L}
$$

In reality, the intensity on the screen is digitized and therefore automatically binned. See Figure 2 (and Figure 12). From each bin, we can calculate the divergence using (4). The range of divergences will be discrete from $M$ bins and we label them as $x_{s 1}^{\prime}, x_{s 2}^{\prime}, \ldots$, $x_{s M}^{\prime}$. For $P$ slits, we can fill these values and their slit positions with intensity values $n_{11}, n_{12}, \ldots, n_{P M}$ into a table. See Table 1.

\begin{tabular}{c|c|c|c|c|c|l}
\hline \multicolumn{1}{c}{ Table 1. Correlation Table } \\
\hline & $\boldsymbol{x}_{\boldsymbol{s} \mathbf{1}}^{\prime}$ & $\boldsymbol{x}_{\boldsymbol{s} \mathbf{2}}^{\prime}$ & $\boldsymbol{x}_{\boldsymbol{s} \mathbf{3}}^{\prime}$ & $\ldots$ & $\boldsymbol{x}_{\boldsymbol{s} \boldsymbol{M}}^{\prime}$ & Total \\
\hline $\boldsymbol{x}_{\boldsymbol{s} \mathbf{1}}$ & $n_{11}$ & $n_{12}$ & $n_{13}$ & $\ldots$ & $n_{1 M}$ & $N_{1}^{\prime}=\sum_{i=1}^{M} n_{1 i}$ \\
$\boldsymbol{x}_{\boldsymbol{s} \mathbf{2}}$ & $n_{21}$ & $n_{22}$ & $n_{23}$ & $\ldots$ & $n_{2 M}$ & $N_{2}^{\prime}=\sum_{i=1}^{M} n_{2 i}$ \\
$\boldsymbol{x}_{\boldsymbol{s} \mathbf{3}}$ & $n_{31}$ & $n_{32}$ & $n_{33}$ & $\ldots$ & $n_{1 M}$ & $N_{3}^{\prime}=\sum_{i=1}^{M} n_{3 i}$ \\
$\vdots$ & $\vdots$ & $\vdots$ & $\vdots$ & $\vdots$ & $\vdots$ & $\vdots$ \\
$\boldsymbol{x}_{\boldsymbol{s} \boldsymbol{P}}$ & $n_{P 1}$ & $n_{P 2}$ & $n_{P 3}$ & $\ldots$ & $n_{P M}$ & $N_{P}^{\prime}=\sum_{i=1}^{M} n_{P i}$ \\
\hline Total & $N_{1}=$ & $N_{2}=$ & $N_{3}=$ & $\ldots$ & $N_{M}=$ & \\
& $\sum_{i=1}^{P} n_{i 1}$ & $\sum_{i=1}^{P} n_{i 2}$ & $\sum_{i=1}^{P} n_{i 3}$ & $\ldots$ & $\sum_{i=1}^{P} n_{i M}$ & \\
\hline \hline
\end{tabular}


We can calculate the following parameters from using the values in Table 1.

$$
\left.\begin{array}{cc}
\bar{x}_{s j}=\frac{1}{N_{j}} \sum_{i=1}^{P} n_{i j} x_{s i} & \bar{x}_{s j}^{\prime}=\frac{1}{N_{j}^{\prime}} \sum_{i=1}^{M} n_{j i} x_{s i}^{\prime} \\
\sigma_{s x j}^{2}=\frac{1}{N_{j}} \sum_{i=1}^{P} n_{i j}\left(x_{s i}-\bar{x}_{s j}\right)^{2} & \sigma_{s x^{\prime} j}^{2}=\frac{1}{N_{j}^{\prime}} \sum_{i=1}^{M} n_{j i}\left(x_{s i}^{\prime}-\bar{x}_{s j}^{\prime}\right)^{2} \\
\bar{\sigma}_{s x}=\frac{1}{M} \sum_{j=1}^{M} \sigma_{s x j} & \bar{\sigma}_{s x^{\prime}}=\frac{1}{P} \sum_{j=1}^{P} \sigma_{s x^{\prime} j}
\end{array}\right\}
$$

These values will be used as input parameters for calculating emittance in the Theory section.

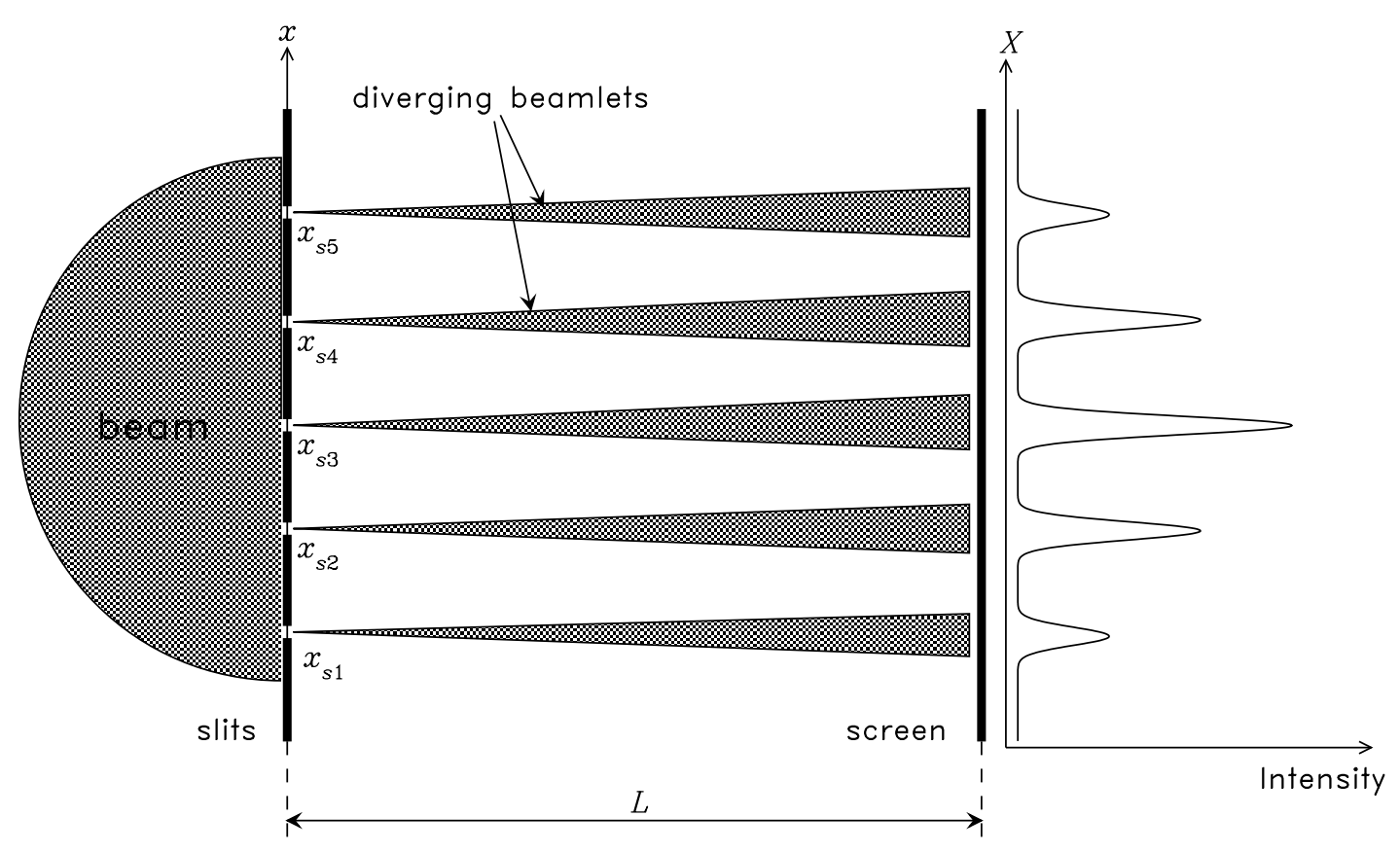

Figure 1 This is the usual setup for measuring emittance with slits. The distance between the slits and screen is $L$. The position of the slits $x_{s 1}, x_{s 2}, \ldots$ are known. The $X$ labels the position of the beamlets on the screen. In this picture, we have only shown 5 slits. 

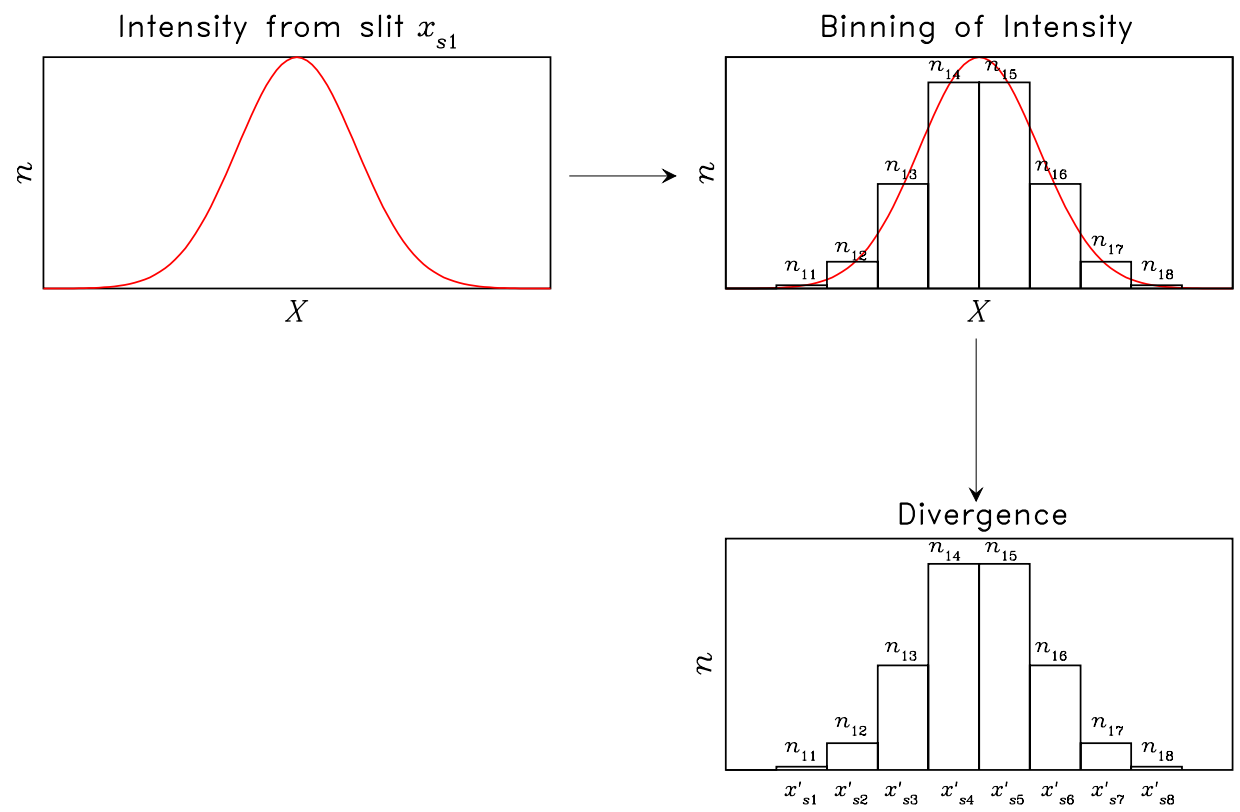

Figure 2 This shows the binning process. The particle intensity from slit $x_{s 1}$ on the screen is captured by a digital camera. Each pixel on the camera integrates the intensity to give a number $n_{1 j}$. The divergence $x_{s j}^{\prime}$ is calculated from the position data using (4).

\section{THEORY}

We start by examining the phase space distribution in $x x^{\prime}$ space for $N$ particles. When this distribution is projected onto the $x$ and $x^{\prime}$ axis, we obtain the frequency distribution of the particles in $x$ and $x^{\prime}$. See Figure 3. Clearly, we have to make some assumptions about the distributions because in general, there are no constraints on it. Thus, to make the problem tractable, we will assume (and hope that the assumptions are indeed reasonable) that the projections onto each axes are independent and the distributions are Gaussian. We will examine later the cases when the projected distributions are not Gaussian.

Once the Gaussian assumption is made, we can write down the normalized frequency 


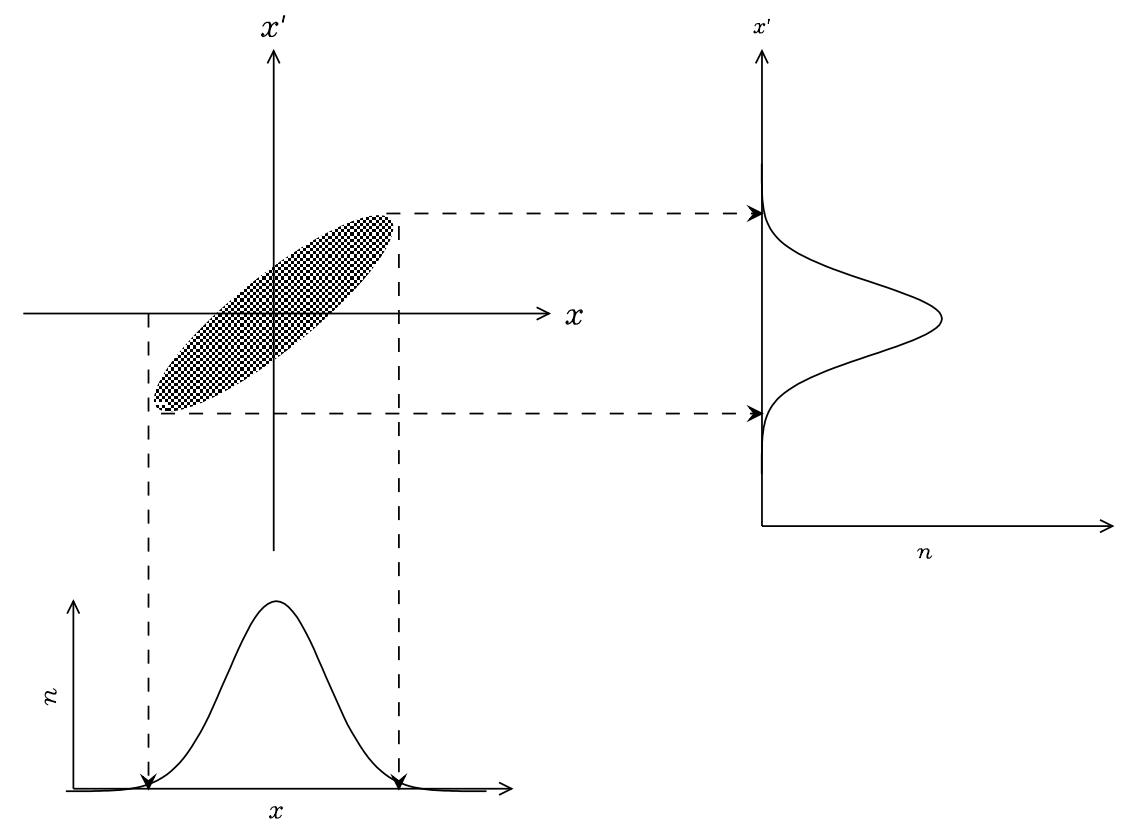

Figure 3 The $x x^{\prime}$ phase space can be projected onto the $x$ and $x^{\prime}$ axes which gives us the frequency distribution.

distribution $\rho_{x}$ of $x$ and $\rho_{x^{\prime}}$ of $x^{\prime}$

$$
\left.\begin{array}{c}
\rho_{x}(x)=\frac{1}{\sqrt{2 \pi} \sigma_{x}} e^{-\frac{(x-a)^{2}}{2 \sigma_{x}^{2}}} \\
\rho_{x^{\prime}}\left(x^{\prime}\right)=\frac{1}{\sqrt{2 \pi} \sigma_{x^{\prime}}} e^{-\frac{\left(x^{\prime}-b\right)^{2}}{2 \sigma_{x^{\prime}}^{2}}}
\end{array}\right\}
$$

where $a=\sum_{i=1}^{N} x_{i} / N$ is the mean position of the $x$ distribution and $b=\sum_{i=1}^{N} x_{i}^{\prime} / N$ is the mean position of the $x^{\prime}$ distribution, $\sigma_{x}=\sqrt{\sum_{i=1}^{N}(x-a)^{2} / N}$ is the standard deviation of the projected $x$ distribution, $\sigma_{x^{\prime}}=\sqrt{\sum_{i=1}^{N}\left(x^{\prime}-b\right)^{2} / N}$ is the standard deviation of the projected $x^{\prime}$ distribution.

$\phi\left(x, x^{\prime}\right)$ is thus (See Appendix I)

$$
\phi\left(x, x^{\prime}\right)=\frac{\exp \left(-\frac{1}{2\left(1-r^{2}\right)}\left[\frac{(x-a)^{2}}{\sigma_{x}^{2}}-\frac{2 r(x-a)\left(x^{\prime}-b\right)}{\sigma_{x} \sigma_{x^{\prime}}}+\frac{\left(x^{\prime}-b\right)^{2}}{\sigma_{x^{\prime}}^{2}}\right]\right)}{2 \pi \sigma_{x} \sigma_{x^{\prime}} \sqrt{1-r^{2}}}
$$


where $r=\sum_{i=1}^{N}\left(x_{i}-a\right)\left(x_{i}^{\prime}-b\right) /\left(N \sigma_{x} \sigma_{x}^{\prime}\right)$ is the coefficient of correlation. For a phase space ellipse that is not tilted, $r=0$.

For the slits experiment, we sample the beam and thus do not have access to $\left(x, x^{\prime}\right)$ for every particle. Thus, we have to come up with a way to calculate $a, b, \sigma_{x}, \sigma_{x^{\prime}}$ and $r$ from this sampled subset.

\section{Slits}

Let us suppose there are $P$ infinitesimally wide slits and that the position of the slits is known exactly. This implies that the position of the beamlets is also known exactly. Let us name the slit positions as $x_{s 1}, x_{s 2}, \ldots, x_{s P}$. Since the beamlet positions are known exactly, the normalized distribution $f(x)$ of $x$ at slit $x_{s j}$ is simply

$$
f\left(x_{s j}\right)=\left.\int_{-\infty}^{\infty} d x^{\prime} \phi\left(x, x^{\prime}\right)\right|_{x=x_{s j}}=\frac{1}{\sqrt{2 \pi} \sigma_{x}} e^{-\frac{\left(x_{s j}-a\right)^{2}}{2 \sigma_{x}^{2}}}
$$

which is exactly what we would have expected from (6). Therefore, the normalized distribution $g\left(x_{s j}, x^{\prime}\right)$ can be found from (1)

$$
g\left(x_{s j}, x^{\prime}\right)=\frac{\phi\left(x_{s j}, x^{\prime}\right)}{f\left(x_{s j}\right)}=\frac{\exp \left(-\frac{1}{2\left(1-r^{2}\right) \sigma_{x^{\prime}}^{2}}\left[x^{\prime}-b-\frac{\sigma_{x^{\prime}} r\left(x_{s j}-a\right)}{\sigma_{x}}\right]^{2}\right)}{\sigma_{x^{\prime}} \sqrt{2 \pi\left(1-r^{2}\right)}}
$$

Clearly, $g\left(x_{s j}, x^{\prime}\right)$ is Gaussian and the beamlet from slit $x_{s j}$ has a mean divergence

$$
\bar{x}_{s j}^{\prime}=b+\frac{\sigma_{x^{\prime}} r}{\sigma_{x}}\left(x_{s j}-a\right)
$$

and standard deviation

$$
\sigma_{s x^{\prime}}=\sigma_{x^{\prime}} \sqrt{1-r^{2}}
$$

which is independent of slit position $x_{s j}$. It is interesting to note that if $r=0$, i.e. $g\left(x, x^{\prime}\right)$ is independent of $x$, both the mean and the standard deviation of the divergence of each beamlet are equal to the mean and standard deviation of the divergence of the beam. 
If we plot $\bar{x}_{s j}^{\prime}$ versus $x_{s j}$ for $j=1, \ldots, P$, we can fit a straight line through these points. If we let $m_{x}$ and $c_{x}$ be the slope and intercept of this line, then immediately

$$
m_{x}=\frac{\sigma_{x^{\prime}} r}{\sigma_{x}} \quad \text { and } \quad c_{x}=b-m_{x} a
$$

The standard deviation of the divergence from each beamlet should be identical and so an average of them $\bar{\sigma}_{s x^{\prime}}$ can be taken to represent $\sigma_{s x^{\prime}}$.

A similar argument can be made for knowing $x_{s i}^{\prime}$ exactly. Especially, since $x_{s i}^{\prime}$ can be thought of as the calculated divergence from each pixel on the screen. Then the relationship between mean position $\bar{x}_{s i}$ and the divergence $x_{s i}^{\prime}$ is

$$
x_{s i}^{\prime}-b=\frac{\sigma_{x^{\prime}}}{\sigma_{x} r}\left(\bar{x}_{s i}-a\right)
$$

where $i=1,2, \ldots, M$ and $M$ is the number of pixels in the $x$ direction. The standard deviation of this distribution is similarly independent of $x_{s i}^{\prime}$ and is

$$
\sigma_{s x}=\sigma_{x} \sqrt{1-r^{2}}
$$

Doing the same thing as before, we can plot $x_{s i}^{\prime}$ versus $\bar{x}_{s i}$ for $i=1,2, \ldots, M$ and fit a straight line through these points. Let the slope of the line be $m_{x^{\prime}}$ and intercept be $c_{x^{\prime}}$, then

$$
m_{x^{\prime}}=\frac{\sigma_{x^{\prime}}}{\sigma_{x} r} \quad \text { and } \quad c_{x^{\prime}}=b-m_{x^{\prime}} a
$$

The average of all the standard deviations $\bar{\sigma}_{s x}$ of each beamlet must be equal to (14).

Summarizing, we have the following equations to solve for $a, b, \sigma_{x}, \sigma_{x^{\prime}}$ and $r$

$$
\left.\begin{array}{lcl}
m_{x}=\frac{\sigma_{x^{\prime}} r}{\sigma_{x}} & c_{x}=b-m_{x} a \\
m_{x^{\prime}}=\frac{\sigma_{x}}{\sigma_{x} r} & c_{x^{\prime}}=b-m_{x^{\prime}} a \\
\sigma_{x^{\prime}}=\sigma_{x^{\prime}} \sqrt{1-r^{2}} & \bar{\sigma}_{s x}=\sigma_{x} \sqrt{1-r^{2}}
\end{array}\right\}
$$

Since we have six equations and five unknowns, there are no exact solutions! In practice, if the underlying distribution is Gaussian, $\bar{\sigma}_{s x}$ can be very noisy because usually the number slits $P$ is small, and thus we really have five equations to solve. See Example 2. 
For non-Gaussian distributions, (10), (11), (13) and (14) will, in general, not be satisfied. Thus strong deviations from straight lines will indicate that our Gaussian assumption is wrong. However, we can still calculate an effective rms emittance which characterizes the emittance of the parent distribution. See section Non-Gaussian Distributions. 


\section{EXAMPLE 1}

In this example, we have generated a non-tilted bi-gaussian ellipse (See Figure 4) that contains $10^{5}$ particles with $\sigma_{x}=1$ units and $\sigma_{x^{\prime}}=10^{-3}$. Five slits are at positions $x_{s j}=\{-1.5,-0.5,0.5,1.5,2.5\}$ with width of 0.1 units. We assume that there are 20 bins that integrate the intensity on the screen. Thus there are 20 bins which correspond to $\left\{x_{s 1}^{\prime}, x_{s 2}^{\prime}, \ldots, x_{s 20}^{\prime}\right\}$. The $x^{\prime}$ distribution for each slit is shown in Figure 5 which clearly shows that the distributions are Gaussian.

Using the formulæ from (5), we can calculate the $\bar{x}_{s j}^{\prime}$ for each slit and plot it for each slit position $x_{s j}$. For this non-tilted bi-Gaussian phase space distributions, the fit shows that

$$
m_{x}=9 \times 10^{-6} \quad \text { and } \quad c_{x}=-0.1 \times 10^{-4}
$$

which essentially says that $r=0$, i.e. the phase space ellipse is not tilted. The practical criterion for deciding whether phase space ellipse is tilted or not is to compare the excursion of the mean $\bar{x}_{s j}^{\prime}$ to the mean standard deviation $\bar{\sigma}_{s x^{\prime}}$

$$
\left|\frac{\max \left(\bar{x}_{s j}^{\prime}\right)-\min \left(\bar{x}_{s j}^{\prime}\right)}{\bar{\sigma}_{s x^{\prime}}}\right|=\left|\frac{8.5 \times 10^{-6}+1.8 \times 10^{-5}}{0.1 \times 10^{-2}}\right|=0.03 \ll 1
$$

This means that the mean and standard deviation of each beamlet is the same as the full beam mean and standard deviation. The calculated $\sigma_{s j}^{\prime}=\{0.1,0.1,0.1,0.1,0.1\} \times 10^{-2}$ which is exactly the standard deviation of the divergence of the full distribution.

We can perform the procedure for calculating $\sigma_{x}$ by using the $r=0$ criterion. Then we see that $\bar{\sigma}_{s x}=1$ if we use the columns of the correlation table which are full, i.e. no zeros. See Figure 6. Note that there are only five points per curve because we only have five slits. 


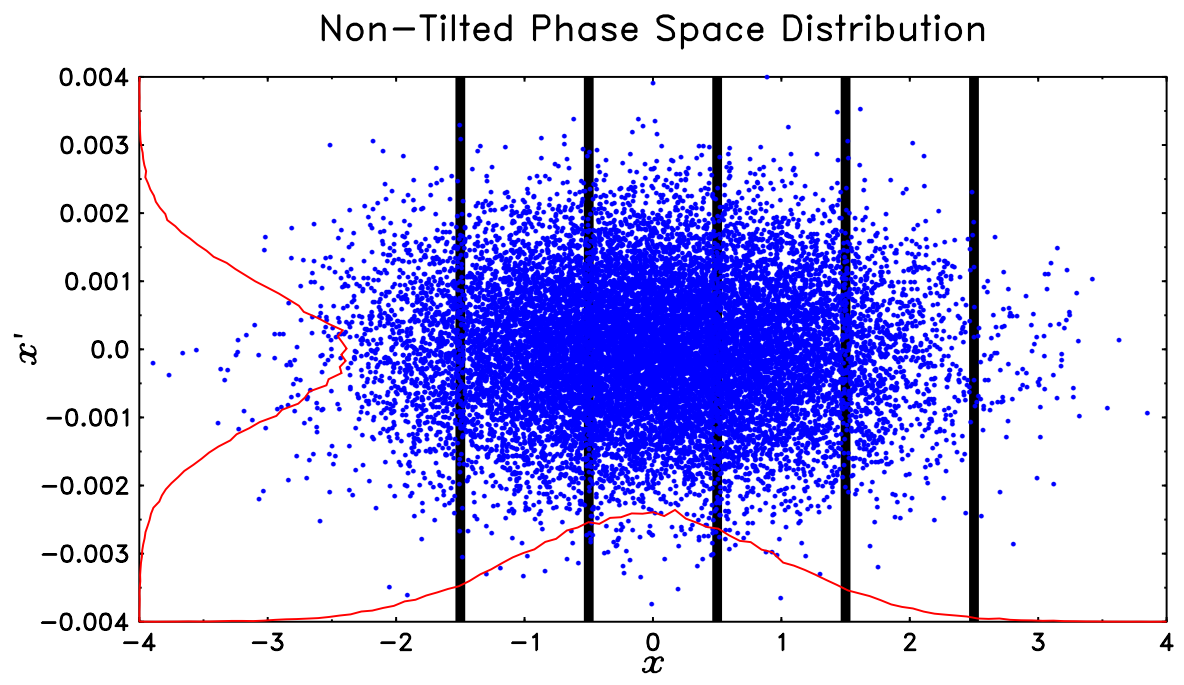

Figure 4 The distribution of this non-tilted phase space is projected on both the $x$ and $x^{\prime}$ axes shown in red. This phase space has $\sigma_{x}=1$ and $\sigma_{x^{\prime}}=10^{-3}$. The slits at $-1.5,-0.5,0.5,1.5,2.5$ (shown as thick black bars) select out the particles with $x^{\prime}$ shown in Figure 5 . 


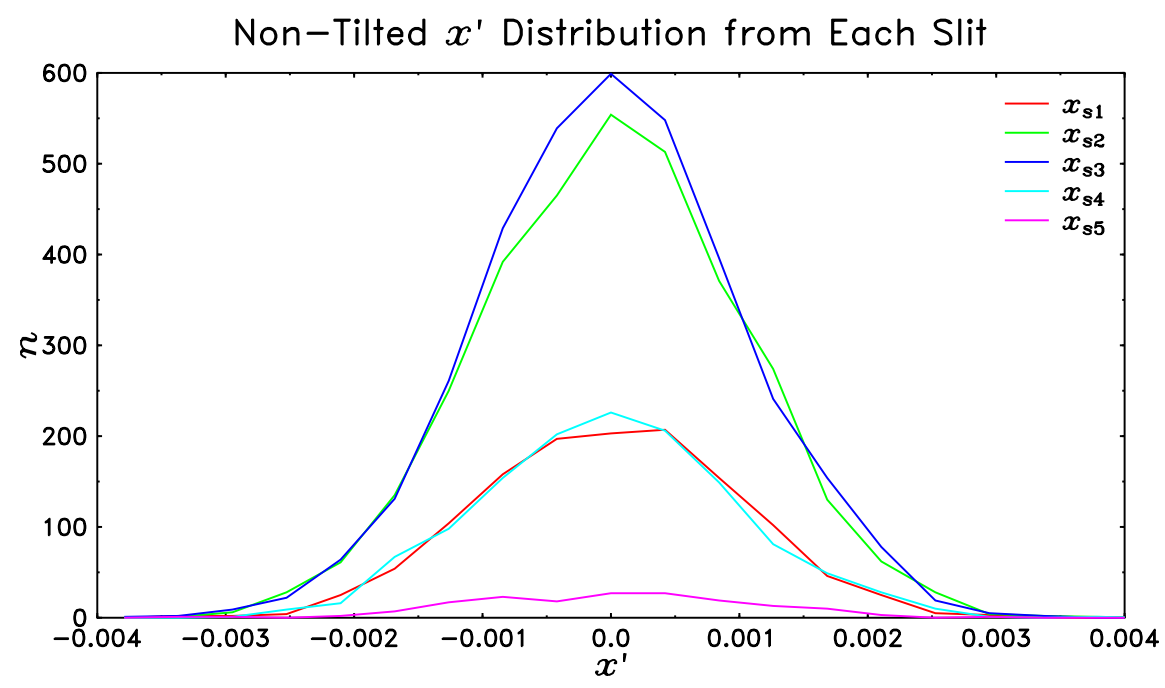

Figure 5 The distribution from each slit is shown here. The curves, which clearly look Gaussian, are formed after binning the $x^{\prime}$ data from each slit into 20 bins.

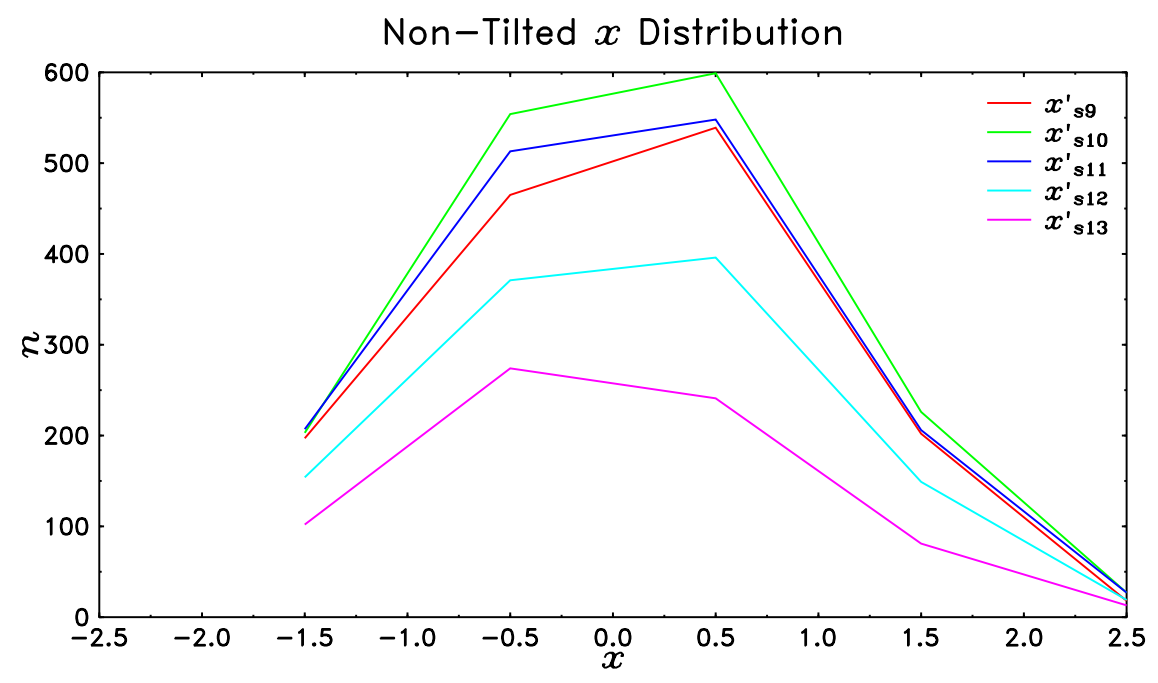

Figure 6 The selected $x$ distributions for $x_{s j}^{\prime}$ where $j=9,10,11,12,13$ for calculating $\bar{\sigma}_{s x}$. 


\section{EXAMPLE 2}

In this example, we have generated a tilted bi-Gaussian ellipse (See Figure 7) that contains $10^{5}$ particles with $\sigma_{x}=1.4$ units and $\sigma_{x^{\prime}}=0.1 \times 10^{-2}$ and $\left\langle x x^{\prime}\right\rangle=0.1 \times 10^{-2}$. The rms emittance calculated using (2) is

$$
\epsilon=0.1 \times 10^{-2}
$$

Again, five slits are at positions $x_{s j}=\{-1.5,-0.5,0.5,1.5,2.5\}$ with width of 0.1 units and there are 20 bins that integrate the intensity on the screen. Figure 8 shows the $x^{\prime}$ distribution of the particles from each slit on the screen.

Using the formulæ from (5), we can calculate the $\bar{x}_{s j}^{\prime}$ for each slit and plot it for each slit position $x_{s j}$. For this tilted bi-Gaussian phase space distributions, the fit shows that

$$
m_{x}=(0.50 \pm 0.04) \times 10^{-3} \quad \text { and } \quad c_{x}=(-9 \pm 7) \times 10^{-6}
$$

Notice the large error in $c_{x}$ because $c_{x} \approx 0$. Fortunately, $c_{x}$ is only used for calculating $a$ and $b$ and does not affect the solution for the emittance. For each slit $\sigma_{s x^{\prime} j}$ is expected to be constant, and indeed we see that $\bar{\sigma}_{s x^{\prime}}=(0.72 \pm 0.01) \times 10^{-3}$.

For amusement, let us check the tilt criterion from (18)

$$
\left|\frac{\max \left(\bar{x}_{s j}^{\prime}\right)-\min \left(\bar{x}_{s j}^{\prime}\right)}{\bar{\sigma}_{s x^{\prime}}}\right|=\left|\frac{0.1 \times 10^{-2}+0.07 \times 10^{-2}}{0.07 \times 10^{-2}}\right|=2.4>1
$$

which tells us that we have a tilted ellipse.

If we plot $x_{s i}^{\prime}$ versus $\bar{x}_{s i}$ after selecting out columns in the correlation table that have no zero entries (See Figure 9), we find that

$$
m_{x^{\prime}}=(0.114 \pm 0.004) \times 10^{-2} \quad \text { and } \quad c_{x^{\prime}}=(-6 \pm 5) \times 10^{-5}
$$

Again, $c_{x^{\prime}}$ has a large error because it is approximately zero. We will not calculate $\sigma_{s x i}$ because of the small number points for each $x_{s i}^{\prime}$. And so we have five equations and five unknowns if we leave $\bar{\sigma}_{s x}$ out, i.e. (16) has a unique solution. 
Substituting the values $m_{x}, c_{x}, \sigma_{s x^{\prime}}, m_{x^{\prime}}$ and $c_{x^{\prime}}$ found above into (16), we find that

$$
\begin{aligned}
& \sigma_{x}=1.3 \pm 0.1 \quad \sigma_{x^{\prime}}=(0.96 \pm 0.01) \times 10^{-3} \\
& r=(0.66 \pm 0.02) \quad \Rightarrow \quad\left\langle x x^{\prime}\right\rangle=r \sigma_{x} \sigma_{x^{\prime}}=(0.8 \pm 0.1) \times 10^{-3}
\end{aligned}
$$

The emittance $\epsilon$ from these values is

$$
\epsilon=(0.09 \pm 0.01) \times 10^{-2}
$$

We can compare these values found by the method of correlation (22) to the parameters used to create the tilt phase space and we see that they agree quite well. $a$ and $b$ have not been explicitly calculated but it is obvious that both are approximately zero in spite of the large uncertainties in $c_{x}$ and $c_{x^{\prime}}$.

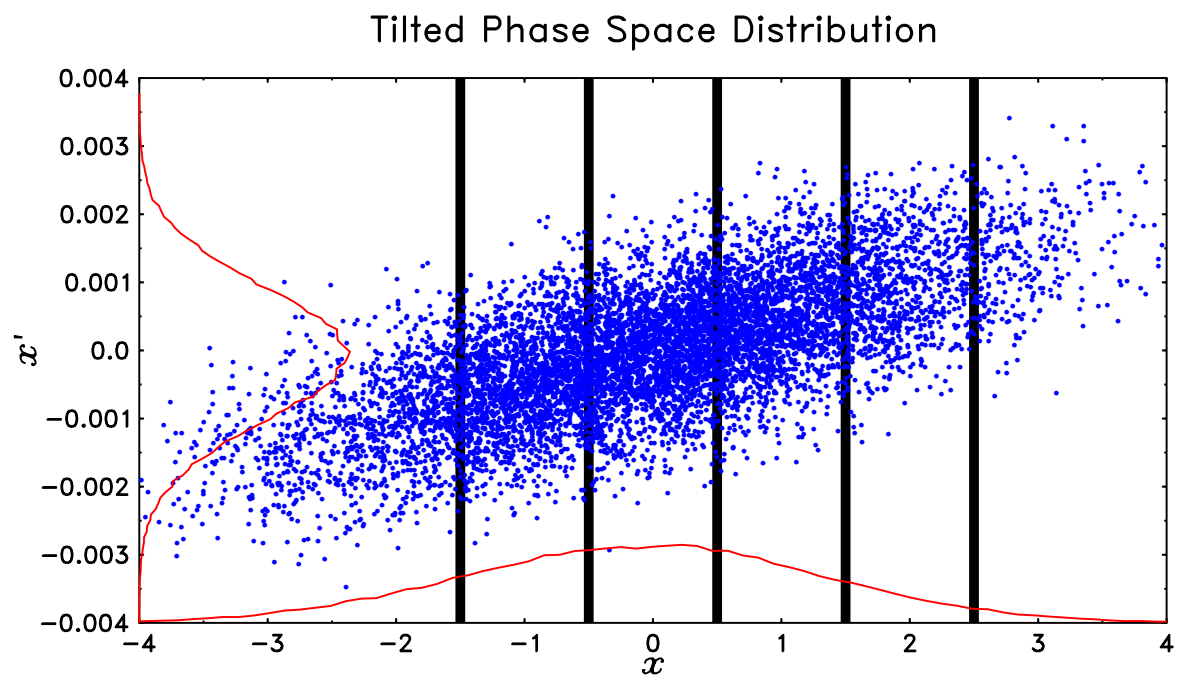

Figure 7 The distribution of this tilted phase space is projected on both the $x$ and $x^{\prime}$ axes shown in red. This phase space has $\sigma_{x}=1.4$ and $\sigma_{x^{\prime}}=0.7 \times 10^{-3}$ and $\left\langle x x^{\prime}\right\rangle=0.1 \times 10^{-2}$. The slits at $-1.5,-0.5,0.5,1.5,2.5$ (shown as thick black bars) select out the particles with $x^{\prime}$ shown in Figure 8. 


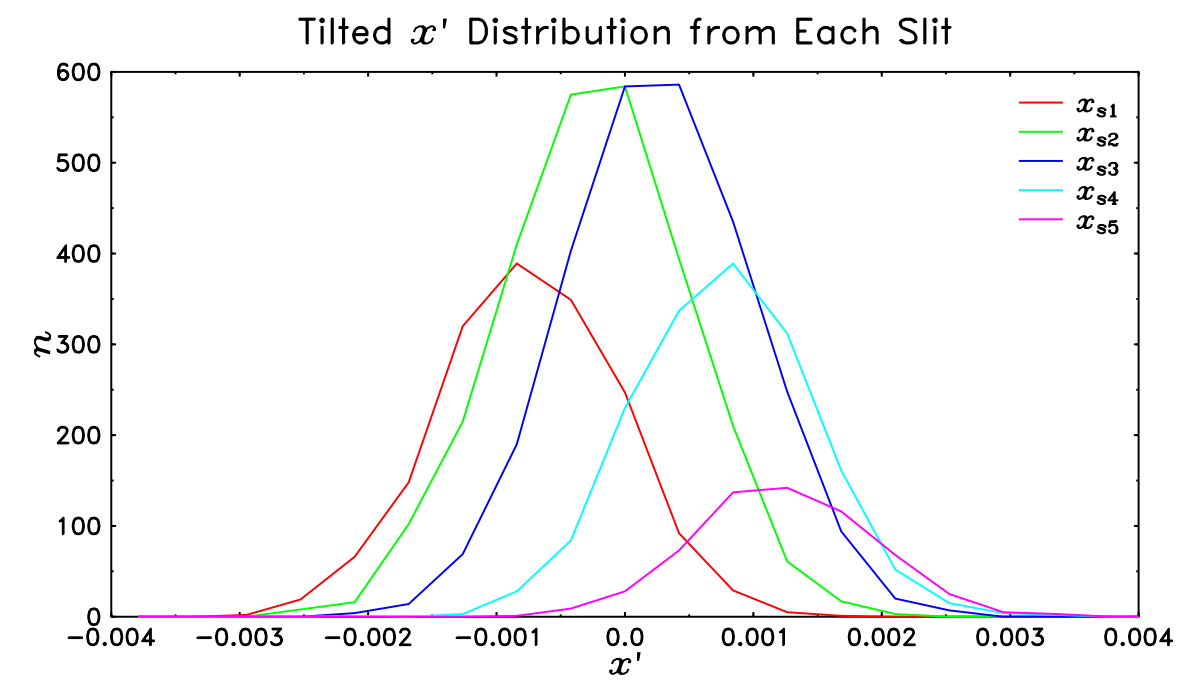

Figure 8 The distribution from each slit is shown here. These curves are formed after binning the $x^{\prime}$ data from each slit into 20 bins. The curves shift with each slit because the phase space distribution is tilted.

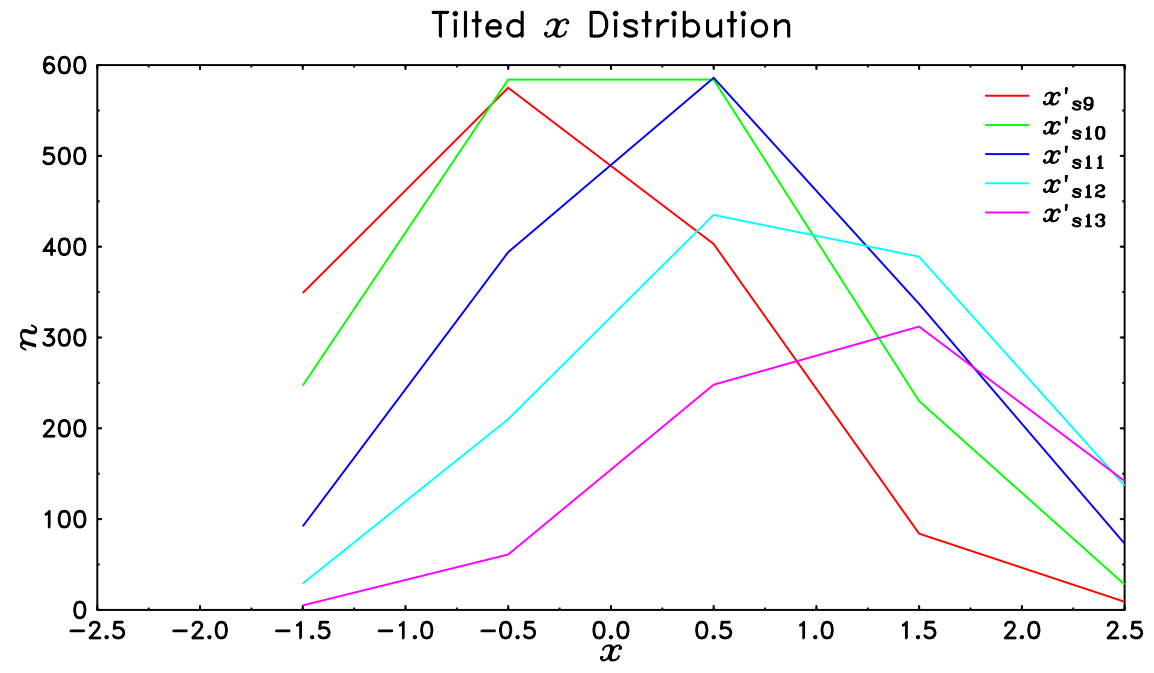

Figure 9 The selected $x$ distributions for $x_{s j}^{\prime}$ where $j=9,10,11,12,13$ for calculating $\bar{\sigma}_{s x}$. 


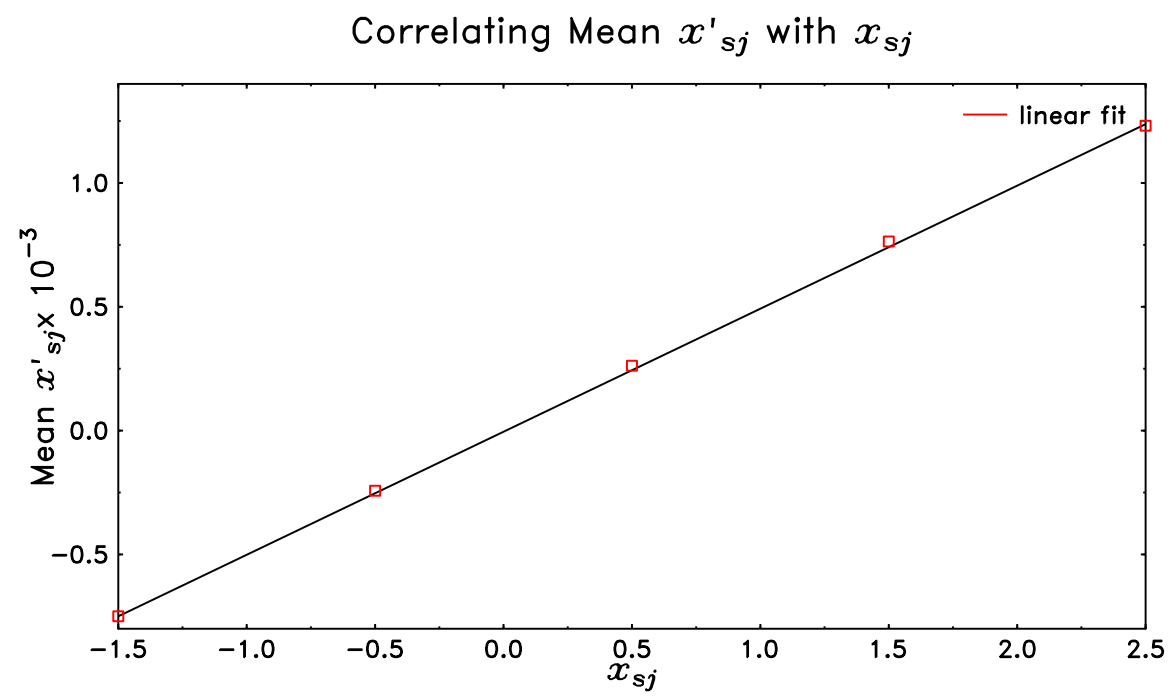

Figure 10 We plot $\bar{x}_{s j}^{\prime}$ versus $x_{s j}$. We expect to see a straight line fit because of (10). The fit parameters are shown in (19).

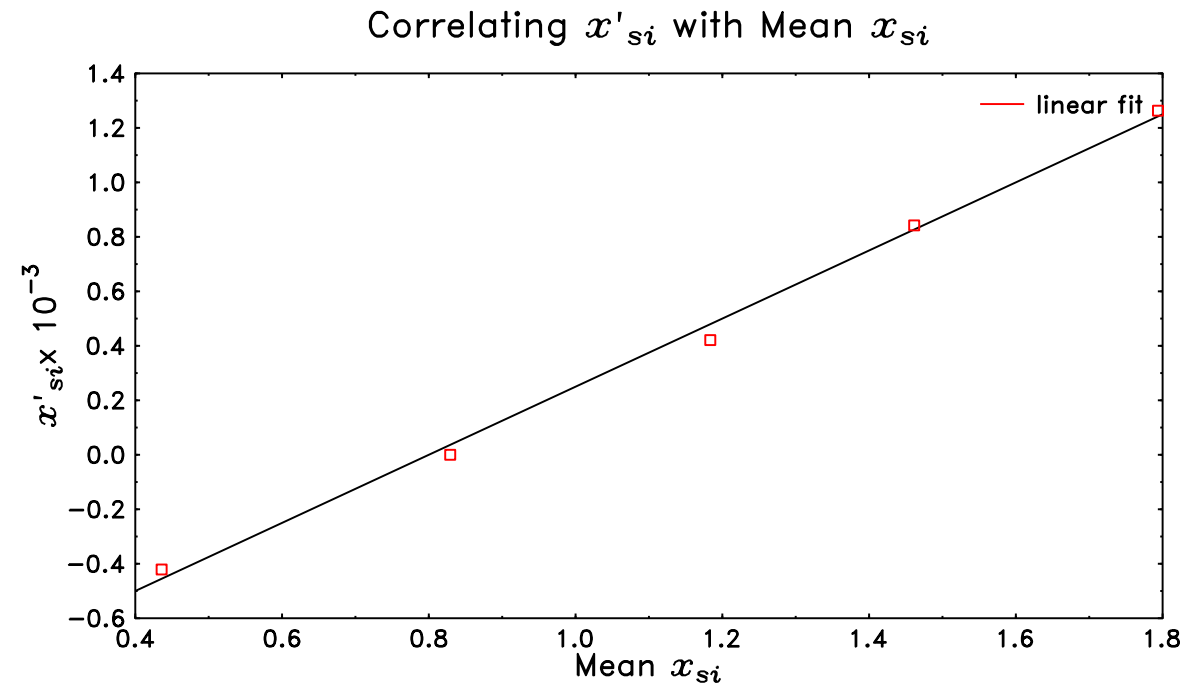

Figure 11 We plot $x_{s i}^{\prime}$ versus $\bar{x}_{s i}$. We expect to see a straight line fit because of (13). The fit parameters are shown in (21). 


\section{NON-GAUSSIAN DISTRIBUTIONS}

As we have stated earlier in the section Theory, for a non-Gaussian distribution, we do not expect the relationship between the mean divergence $\bar{x}_{s j}^{\prime}$ and slit position $x_{s j}$ to lie on a straight line (c.f. (10)). Similarly, we do not expect the relationship between the divergence $x_{s i}^{\prime}$ and the mean position $\bar{x}_{s i}$ to lie on a straight line (c.f. (13)) either. And so, deviations from straight lines will help us identify non-Gaussian distributions. The goodness of fit parameter $\chi^{2}$ which tells us how good a linear fit is can serve as the indicator of how good or bad the Gaussian assumption is. We will use actual data to illustrate the analysis of non-Gaussian distributions.

\section{EXAMPLE 3}

In this examples, we will use the slit image measured by $\mathrm{C}$. Bhat at location X3 in the A0 photoinjector at Fermilab. See Figure 12. The distance between slits is $1 \mathrm{~mm}$, the width of each slit is $50 \mu \mathrm{m}$ and the distance between the slits and the screen is $780 \mathrm{~mm}$. To be consistent with the Theory, we will assume that the width of each slit is infinitesimally small. The intensity from each slit is projected onto the vertical axis to form an intensity plot. Note that in this example, the slits are oriented for measuring the vertical emittance and so we will change our labels $x \rightarrow y, x^{\prime} \rightarrow y^{\prime}$ etc.

We will partition the intensity plot into slices. Each slice is $1 \mathrm{~mm}$ in width and centred at $y=1,2,3, \ldots, 13 \mathrm{~mm}$. By doing this, we can calculate $y^{\prime}$ from each slit. This is shown in Figure 13. Clearly all the curves are not Gaussian: $y_{s 5}$ which has 2 humps, while others like $y_{s 3}$ have tails which do not fall off fast enough. ${ }^{\ddagger}$ Looking at Figure 14, we can clearly see that the parent distribution is not Gaussian. But the points lie close enough to a

$\ddagger$ One way to check the Gaussianness of each curve is to calculate the kurtosis. For Gaussians, the kurtusis is identically equal to 3 . 

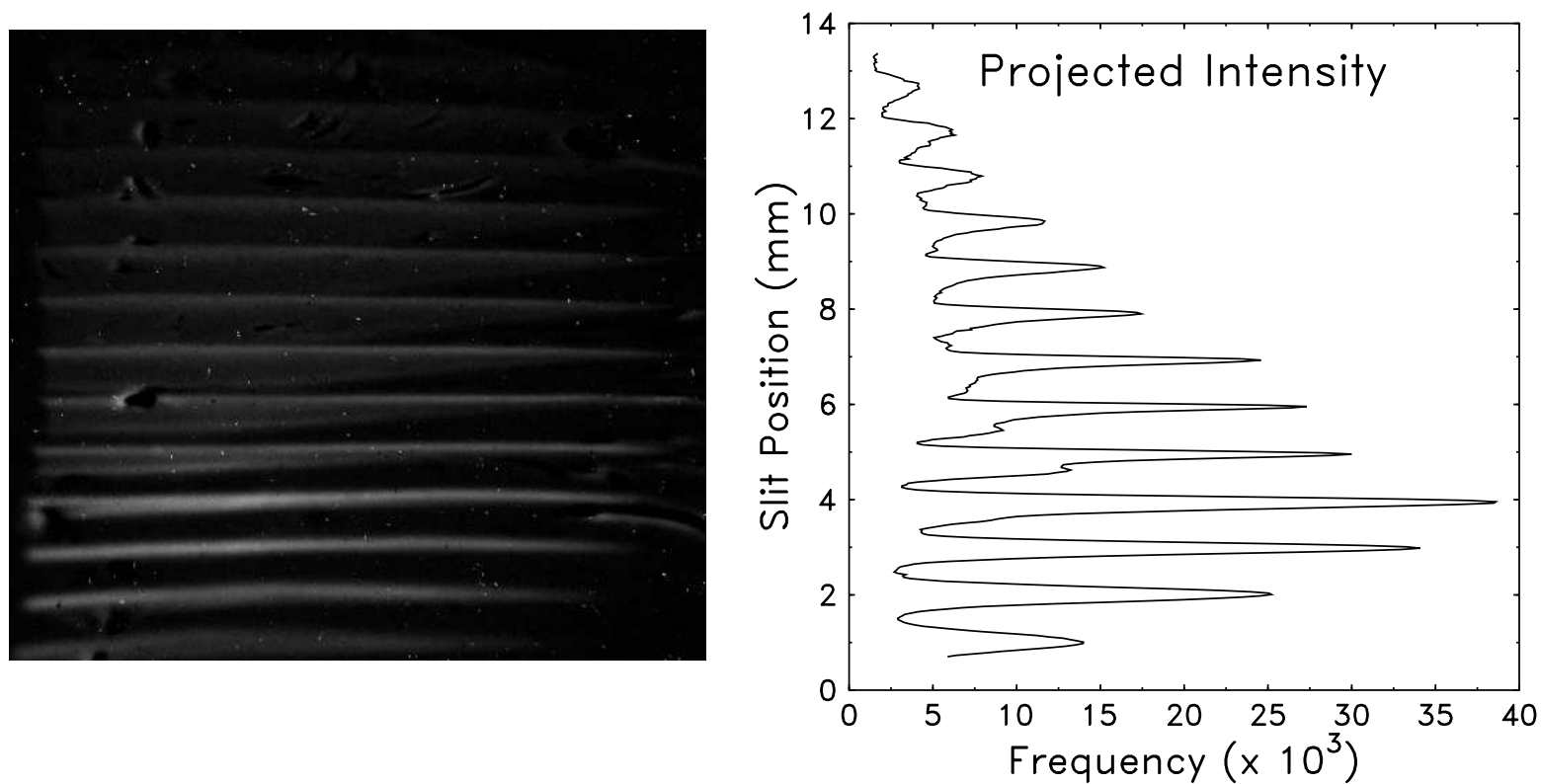

Figure 12 The intensity from each slit on the screen is projected onto the vertical axis to form an intensity plot. The charge in each bunch which produced this image is $2.1 \mathrm{nC}$.

straight line to give the following fit:

$$
m_{x}=(-1.2 \pm 0.3) \times 10^{-5} \mathrm{rad} / \mathrm{mm} \quad c_{x}=(1 \pm 2) \times 10^{-5} \mathrm{rad}
$$

Notice that the error is huge for $c_{x}$. But $c_{x}$ is only used for calculating $a$ and $b$ which does not affect the emittance calculation.

Next we plot out the $\sigma_{s y^{\prime} j}$ to see how the points scatter. See Figure 15. The average $\bar{\sigma}_{s y^{\prime}}$ is

$$
\bar{\sigma}_{s y^{\prime}}=(30 \pm 1) \times 10^{-5} \mathrm{rad}
$$

The plot of $y_{s i}^{\prime}$ versus $\bar{y}_{s i}$ is shown in Figure 16. Again, this confirms the nonGaussianness of the parent distribution. Since, clearly, we cannot use a linear fit for this set of data, we have to see if $\sigma_{s y j}$ can be used to calculate $\bar{\sigma}_{s y}$. See Figure 17 . The $\bar{\sigma}_{s y}$ calculated from this is

$$
\bar{\sigma}_{s y}=(3.74 \pm 0.05)(\mathrm{mm})
$$


Substituting (24), (25) and (26) into (16), we can calculate $\sigma_{y}, \sigma_{y^{\prime}}$ and $r$ (foregoing the calculation of $a$ and $b$ )

$$
\begin{aligned}
\sigma_{y} & =(3.79 \pm 0.05) \mathrm{mm} \\
\sigma_{y^{\prime}} & =(0.31 \pm 0.01) \mathrm{mrad} \\
r & =(-0.15 \pm 0.04) \Rightarrow\left\langle y y^{\prime}\right\rangle=r \sigma_{y} \sigma_{y^{\prime}}=(-0.18 \pm 0.04) \mathrm{mm} \cdot \mathrm{mrad}
\end{aligned}
$$

And thus the effective rms emittance of this non-Gaussian distribution is

$$
\epsilon=(1.2 \pm 0.1) \mathrm{mm} \cdot \mathrm{mrad}
$$

Finally, we can plot the ellipses using the values calculated above and compare them with the sampled phase space from slits. The ellipses are calculated by extracting out the argument in the exponent of $(7)$

$$
\frac{1}{\left(1-r^{2}\right)}\left[\frac{(y-a)^{2}}{\sigma_{y}^{2}}-\frac{2 r(y-a)\left(y^{\prime}-b\right)}{\sigma_{y} \sigma_{y^{\prime}}}+\frac{\left(y^{\prime}-b\right)^{2}}{\sigma_{y^{\prime}}^{2}}\right]=\text { constant } \equiv k
$$

Essentially, this expression allows us to draw out ellipses in terms of " $\sigma$ ", i.e. when $k=1$, this is equivalent to drawing out an ellipse at $1 \sigma$, and when $k=4$, the ellipse is at $2 \sigma$ etc. (Pedantic: For $e^{-x^{2} / 2 \sigma^{2}}$, if $x=\sigma$ then this is equivalent to $k=1$ ).

Using this expression, the ellipses for $1 \sigma$ and $2 \sigma$ are compared with the sampled phase space in Figure 18. We have centred the ellipses at $(4,0)$ because the highest density of particles is at slit 4 from Figure 13. From here, the "effective" emittance which we have calculated covers the expected number of particles from the sampled phase space distribution. 


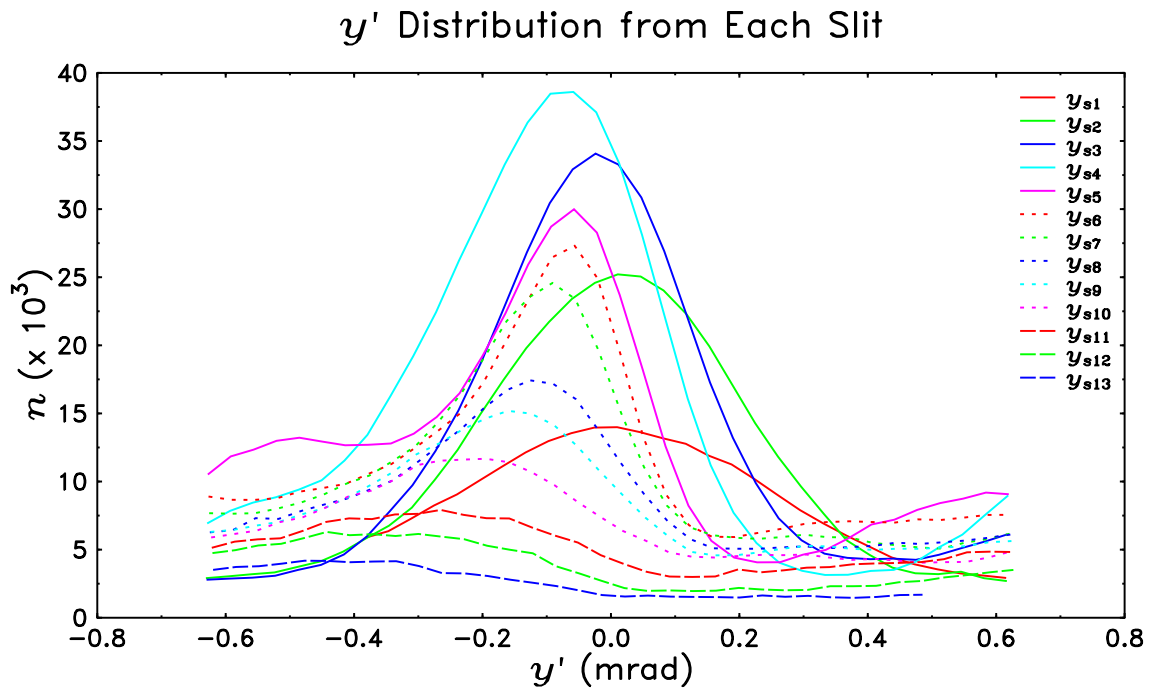

Figure 13 The $y^{\prime}$ distribution from each slit of Figure 12 is shown here.

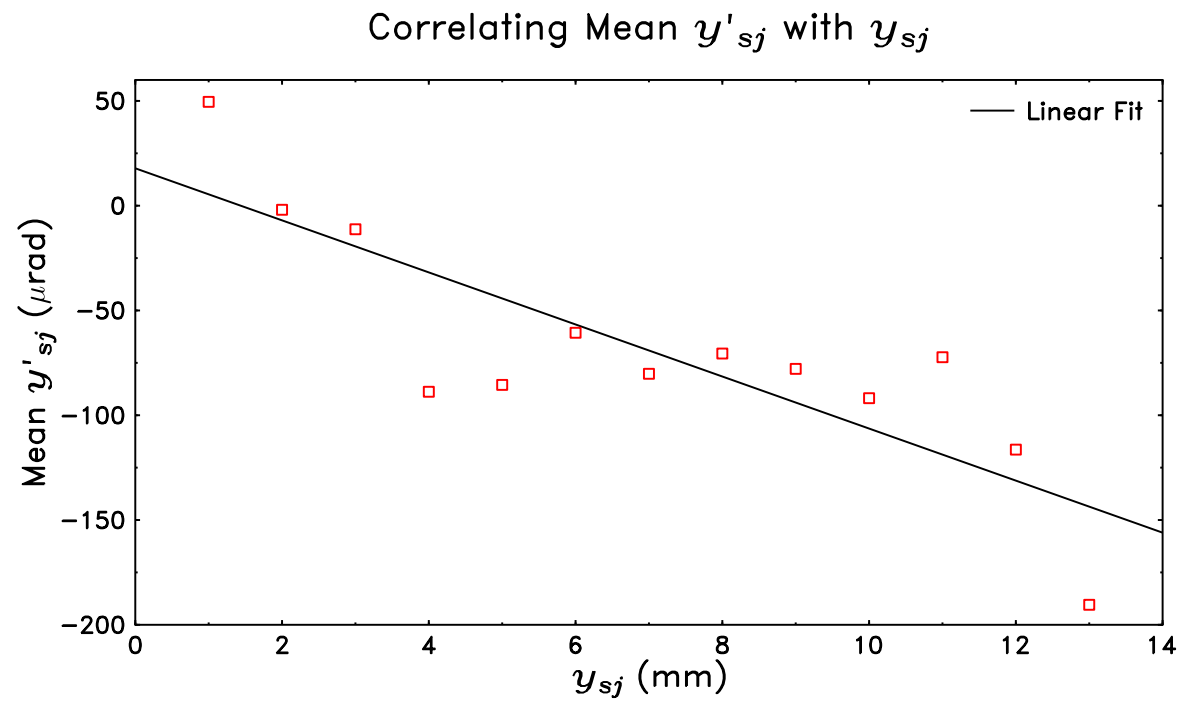

Figure 14 We plot $\bar{y}_{s j}^{\prime}$ versus $y_{s j}$. The parent distribution is not Gaussian but is close enough so that a straight line can be drawn through the points. 


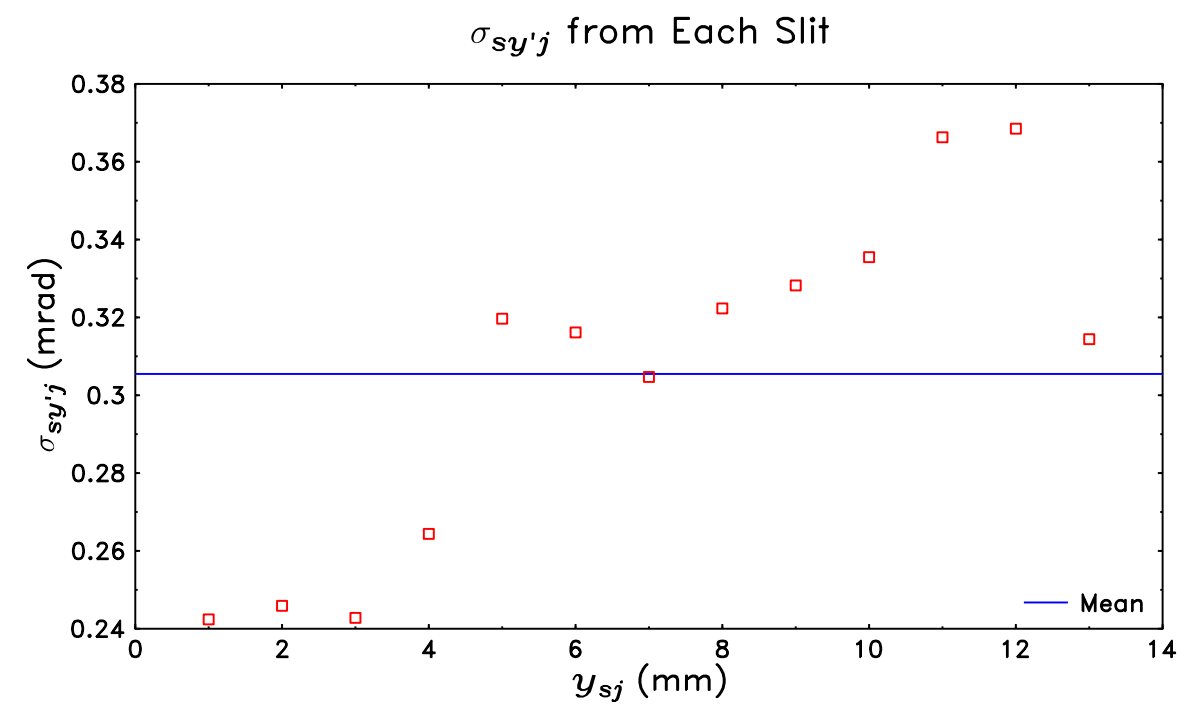

Figure 15 If the parent distribution is Gaussian, $\sigma_{s y^{\prime} j}$ is constant. It is obvious that $\sigma_{s y^{\prime} j}$ is not constant. The blue line drawn here is the average $\bar{\sigma}_{s y^{\prime}}$.

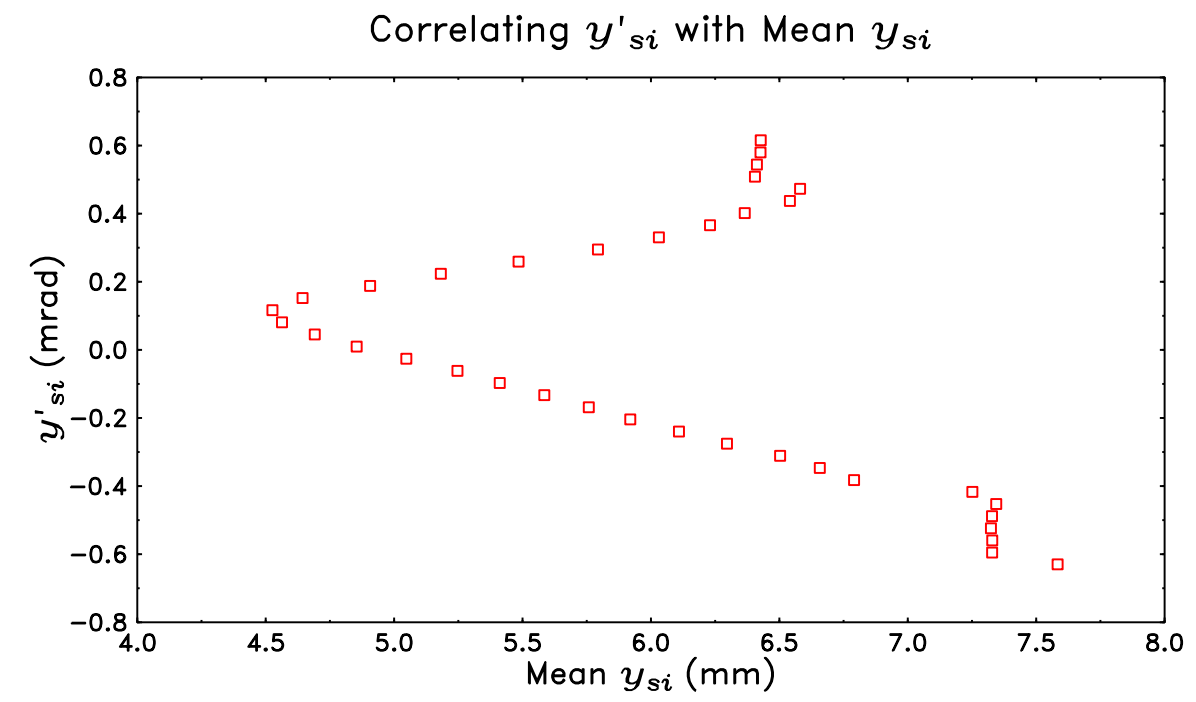

Figure 16 The plot $y_{s i}^{\prime}$ versus $\bar{y}_{s i}$ clearly shows that the points do not lie on a straight line. Therefore, the parent distribution is not Gaussian. 


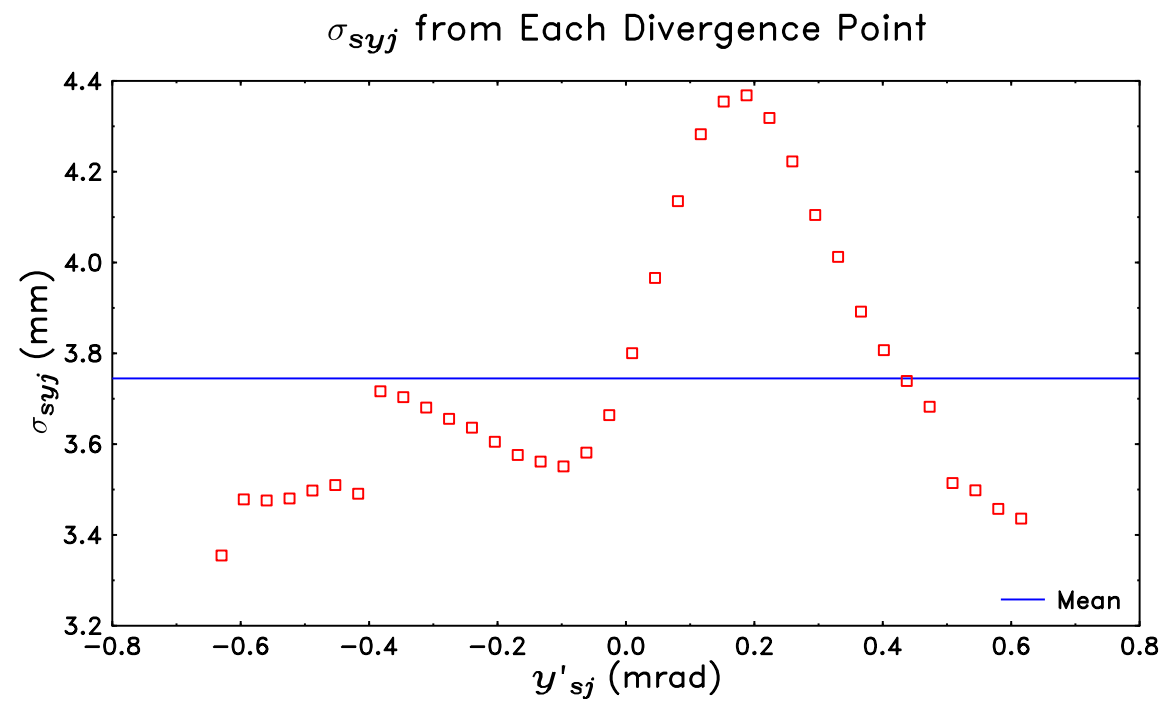

Figure 17 From this plot, we can calculate $\bar{\sigma}_{s y}$ shown as the blue line.

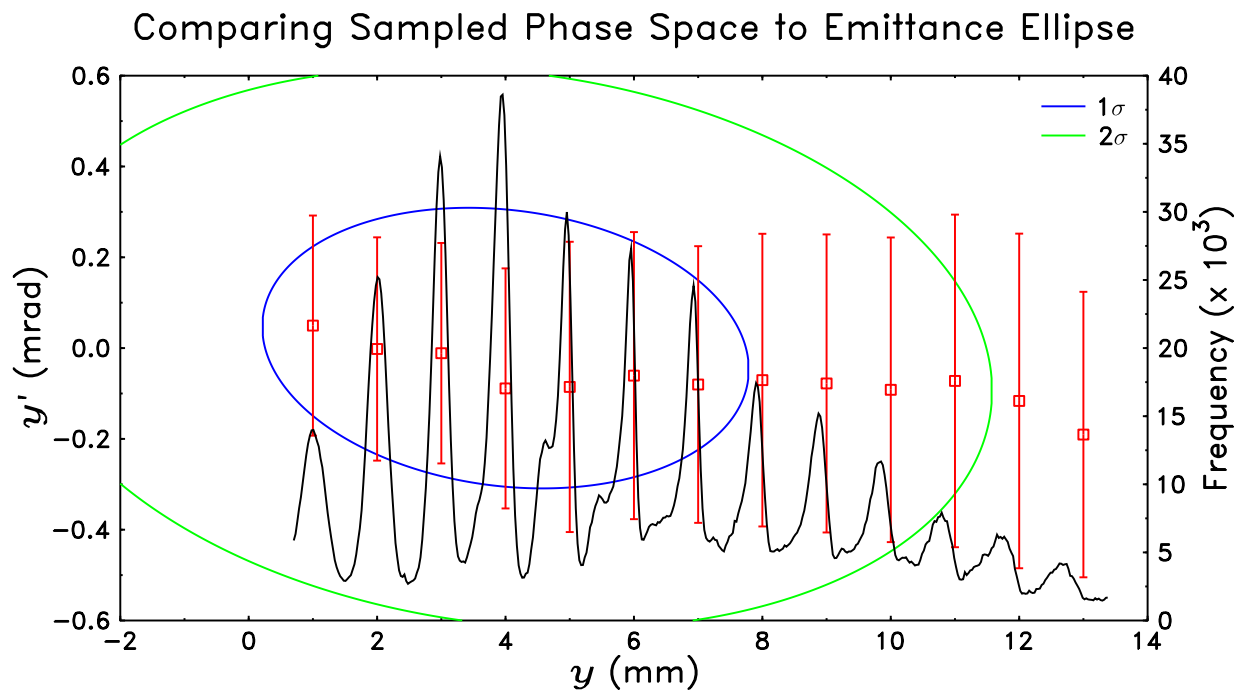

Figure 18 The $1 \sigma$ and $2 \sigma$ contour lines are plotted with the sampled phase space from the slits. The squares are the mean of $y^{\prime}$ and the error bars are the $\pm \sigma_{y^{\prime}}$ from each slit. The projected intensity is also plotted for reference. 


\section{CONCLUSION}

We have shown by examples how the method of correlations can be used to calculate the emittance of the parent distribution. If the parent distribution is Gaussian, this method yields very good results. Even when the parent distribution is non-Gaussian, this method gives an effective emittance that serves as a useful parametrization. Furthermore, statistical errors can be obtained easily from the linear fits, which gives a handle on how good the quoted emittance value is.

\section{ACKNOWLEDGEMENTS}

The author wishes to thank C. Bhat for reading and making suggestions for improving this paper. 


\section{APPENDIX I}

We will prove that the probability function $\phi\left(x, x^{\prime}\right)$ of the bivariate normal distribution is

$$
\phi\left(x, x^{\prime}\right)=\frac{\exp \left(-\frac{1}{2\left(1-r^{2}\right)}\left[\frac{(x-a)^{2}}{\sigma_{x}^{2}}-\frac{2 r(x-a)\left(x^{\prime}-b\right)}{\sigma_{x} \sigma_{x^{\prime}}}+\frac{\left(x^{\prime}-b\right)^{2}}{\sigma_{x^{\prime}}^{2}}\right]\right)}{2 \pi \sigma_{x} \sigma_{x^{\prime}} \sqrt{1-r^{2}}}
$$

Suppose $X$ and $X^{\prime}$ are normally distributed and independent variates with mean 0 and standard deviation 1 respectively. So, if the joint probability density function is $\psi\left(x, x^{\prime}\right)$ then the probability of finding a point which lies between $x+d x$ and $x^{\prime}+d x^{\prime}$ is

$$
\psi\left(x, x^{\prime}\right) d x d x^{\prime}=\frac{1}{2 \pi} e^{-\frac{1}{2}\left(x^{2}+x^{\prime 2}\right)} d x d x^{\prime}
$$

Any other normally distributed variate $Y$ and $Y^{\prime}$ can be created from $X$ and $X^{\prime}$ by an affine transformation, i.e. any point $\left(x, x^{\prime}\right)$ of the $\left(X, X^{\prime}\right)$ distribution is related to $\left(y, y^{\prime}\right)$ of the $\left(Y, Y^{\prime}\right)$ distribution by

$$
\left(\begin{array}{c}
y \\
y^{\prime}
\end{array}\right)=\boldsymbol{T}\left(\begin{array}{c}
x \\
x^{\prime}
\end{array}\right)+\left(\begin{array}{l}
a \\
b
\end{array}\right)
$$

where $\boldsymbol{T}$ is a linear transformation in $\mathbb{R}^{2}$, and $\left(\begin{array}{l}a \\ b\end{array}\right) \in \mathbb{R}^{2}$ is a shift of the origin. For example, the transformation $\boldsymbol{T}$ which relates Example 1 to Example 2 is a scale change followed by a rotation. Therefore, by making a change of variable $\left(x, x^{\prime}\right) \rightarrow\left(y, y^{\prime}\right)$ we can derive the probability density function $\phi\left(y, y^{\prime}\right)$ from (31).

The first step is to solve for $\left(x, x^{\prime}\right)$ in terms of $\left(y, y^{\prime}\right)$. We can do this if $\boldsymbol{T}$ is invertible, or in other words, $\operatorname{det} T \neq 0$, then $\left(\begin{array}{c}x \\ x^{\prime}\end{array}\right)$ from $(32)$ is

$$
\left(\begin{array}{c}
x \\
x^{\prime}
\end{array}\right)=\boldsymbol{T}^{-1}\left(\begin{array}{c}
y-a \\
y^{\prime}-b
\end{array}\right)
$$

If we write $\boldsymbol{T}$ in full matrix form

$$
\boldsymbol{T}=\left(\begin{array}{ll}
t_{11} & t_{12} \\
t_{21} & t_{22}
\end{array}\right)
$$


then

$$
\boldsymbol{T}^{-1}=\frac{1}{t_{11} t_{22}-t_{21} t_{12}}\left(\begin{array}{cc}
t_{22} & -t_{12} \\
-t_{21} & t_{11}
\end{array}\right)
$$

and so

$$
\left(\begin{array}{c}
x \\
x^{\prime}
\end{array}\right)=\frac{1}{t_{11} t_{22}-t_{21} t_{12}}\left(\begin{array}{cc}
t_{22} & -t_{12} \\
-t_{21} & t_{11}
\end{array}\right)\left(\begin{array}{c}
y-a \\
y^{\prime}-b
\end{array}\right)
$$

From (31), we need to calculate $x^{2}+x^{\prime 2}$ in terms of $y$ and $y^{\prime}$. By using (36), we have

$$
x^{2}+x^{\prime 2}=\frac{\left[t_{11}\left(y^{\prime}-b\right)-t_{21}(y-a)\right]^{2}+\left[t_{22}(y-a)-t_{12}\left(y^{\prime}-b\right)\right]^{2}}{\left(t_{11} t_{22}-t_{12} t_{21}\right)^{2}}
$$

We define the following ${ }^{\dagger}$

$$
\left.\begin{array}{c}
\sigma_{y}=t_{11}^{2}+t_{12}^{2} \\
\sigma_{y^{\prime}}=t_{22}^{2}+t_{21}^{2} \\
=\frac{t_{11}+t_{22} t_{12}}{\sigma_{y} \sigma_{y^{\prime}}}
\end{array}\right\}
$$

and substituting them into (37), we find that

$$
x^{2}+x^{\prime 2}=\frac{1}{1-r^{2}}\left[\frac{(y-a)^{2}}{\sigma_{y}^{2}}-\frac{2 r(y-a)\left(y^{\prime}-b\right)}{\sigma_{y} \sigma_{y^{\prime}}}+\frac{\left(y^{\prime}-b\right)^{2}}{\sigma_{y^{\prime}}^{2}}\right]
$$

To transform $d x d x^{\prime} \rightarrow d y d y^{\prime}$, we need the Jacobian

$$
J\left[\frac{x, x^{\prime}}{y, y^{\prime}}\right]=\operatorname{det}\left(\begin{array}{ll}
\frac{\partial x}{\partial y} & \frac{\partial x}{\partial y^{\prime}} \\
\frac{\partial x^{\prime}}{\partial y} & \frac{\partial x^{\prime}}{\partial y^{\prime}}
\end{array}\right)
$$

And with more tedious algebra, we will find that

$$
\left.\begin{array}{rl}
d x d x^{\prime} & =J\left[\frac{x, x^{\prime}}{y, y^{\prime}}\right] d y d y^{\prime} \\
& =\frac{d y d y^{\prime}}{\sigma_{y} \sigma_{y^{\prime}} \sqrt{1-r^{2}}}
\end{array}\right\}
$$

And thus, by substituting (39) and (41) into (31), we get $\phi\left(y, y^{\prime}\right)$.

$\dagger$ with hindsight. 


\section{REFERENCES}

[1] M. Zhang, Emittance Formula for Slits and Pepper-pot Measurement, TM-1988, Fermilab 1996.

[2] E. Whittaker \& G. Robinson, The Calculus of Observations, 4th Edition, Blackie \& Son Ltd, 1960. 